\title{
A Theorem of Fermat on Congruent Number Curves
}

\author{
Lorenz Halbeisen and Norbert Hungerbühler
}

To the memory of S. Srinivasan

\begin{abstract}
A positive integer $A$ is called a congruent number if $A$ is the area of a right-angled triangle with three rational sides. Equivalently, $A$ is a congruent number if and only if the congruent number curve $y^{2}=x^{3}-A^{2} x$ has a rational point $(x, y) \in \mathbb{Q}^{2}$ with $y \neq 0$. Using a theorem of Fermat, we give an elementary proof for the fact that congruent number curves do not contain rational points of finite order.
\end{abstract}

Keywords. congruent numbers, Pythagorean triples.

2010 Mathematics Subject Classification. primary 11G05; secondary 11D25.

\section{Introduction}

A positive integer $A$ is called a congruent number if $A$ is the area of a right-angled triangle with three rational sides. So, $A$ is congruent if and only if there exists a rational Pythagorean tripel $(a, b, c)$ (i.e., $a, b, c \in \mathbb{Q}, a^{2}+b^{2}=c^{2}$, and $a b \neq 0$ ), such that $\frac{a b}{2}=A$. The sequence of integer congruent numbers starts with

$$
5,6,7,13,14,15,20,21,22,23,24,28,29,30,31,34,37, \ldots
$$

For example, $A=7$ is a congruent number, witnessed by the rational Pythagorean triple

$$
\left(\frac{24}{5}, \frac{35}{12}, \frac{337}{60}\right) \text {. }
$$

It is well-known that $A$ is a congruent number if and only if the cubic curve

$$
C_{A}: y^{2}=x^{3}-A^{2} x
$$

has a rational point $\left(x_{0}, y_{0}\right)$ with $y_{0} \neq 0$. The cubic curve $C_{A}$ is called a congruent number curve. This correspondence between rational points on congruent number curves and rational Pythagorean triples can be made explicit as follows: Let

$$
C(\mathbb{Q}):=\left\{(x, y, A) \in \mathbb{Q} \times \mathbb{Q}^{*} \times \mathbb{Z}^{*}: y^{2}=x^{3}-A^{2} x\right\},
$$

where $\mathbb{Q}^{*}:=\mathbb{Q} \backslash\{0\}, \mathbb{Z}^{*}:=\mathbb{Z} \backslash\{0\}$, and

$$
P(\mathbb{Q}):=\left\{(a, b, c, A) \in \mathbb{Q}^{3} \times \mathbb{Z}^{*}: a^{2}+b^{2}=c^{2} \text { and } a b=2 A\right\} .
$$

Then, it is easy to check that

$$
\begin{aligned}
\psi: P(\mathbb{Q}) & \rightarrow C(\mathbb{Q}) \\
(a, b, c, A) & \mapsto\left(\frac{A(b+c)}{a}, \frac{2 A^{2}(b+c)}{a^{2}}, A\right)
\end{aligned}
$$

We thank episciences.org for providing open access hosting of the electronic journal Hardy-Ramanujan Journal 
is bijective and

$$
\begin{aligned}
\psi^{-1}: \quad C(\mathbb{Q}) & \rightarrow P(\mathbb{Q}) \\
(x, y, A) & \mapsto\left(\frac{2 x A}{y}, \frac{x^{2}-A^{2}}{y}, \frac{x^{2}+A^{2}}{y}, A\right) .
\end{aligned}
$$

For positive integers $A$, a triple $(a, b, c)$ of non-zero rational numbers is called a rational Pythagorean $\boldsymbol{A}$-triple if $a^{2}+b^{2}=c^{2}$ and $A=\left|\frac{a b}{2}\right|$. Notice that if $(a, b, c)$ is a rational Pythagorean $A$-triple, then $A$ is a congruent number and $|a|,|b|,|c|$ are the lengths of the sides of a right-angled triangle with area $A$. Notice also that we allow $a, b, c$ to be negative.

It is convenient to consider the curve $C_{A}$ in the projective plane $\mathbb{R} P^{2}$, where the curve is given by

$$
C_{A}: y^{2} z=x^{3}-A^{2} x z^{2} .
$$

On the points of $C_{A}$, one can define a commutative, binary, associative operation "+", where $\mathscr{O}$, the neutral element of the operation, is the projective point $(0,1,0)$ at infinity. More formally, if $P$ and $Q$ are two points on $C_{A}$, then let $P \# Q$ be the third intersection point of the line through $P$ and $Q$ with the curve $C_{A}$. If $P=Q$, the line through $P$ and $Q$ is replaced by the tangent in $P$. Then $P+Q$ is defined by stipulating

$$
P+Q:=\mathscr{O} \#(P \# Q),
$$

where for a point $R$ on $C_{A}, \mathscr{O} \# R$ is the point reflected across the $x$-axis. The following figure shows the congruent number curve $C_{A}$ for $A=5$, together with two points $P$ and $Q$ and their sum $P+Q$.

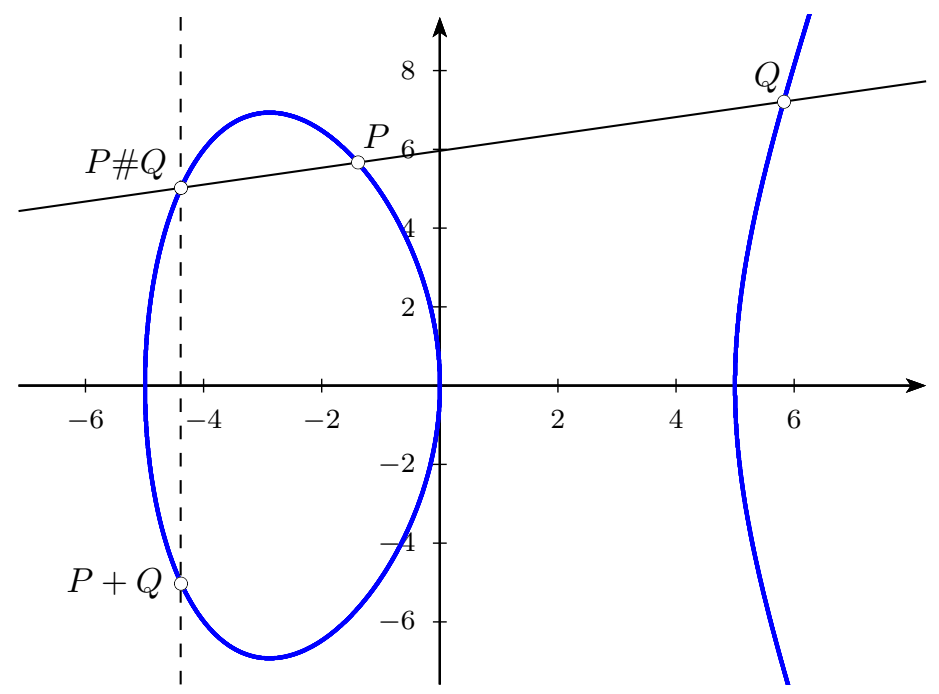

More formally, for two points $P=\left(x_{0}, y_{0}\right)$ and $Q=\left(x_{1}, y_{1}\right)$ on a congruent number curve $C_{A}$, the point $P+Q=\left(x_{2}, y_{2}\right)$ is given by the following formulas:

- If $x_{0} \neq x_{1}$, then

$$
x_{2}=\lambda^{2}-x_{0}-x_{1}, \quad y_{2}=\lambda\left(x_{0}-x_{2}\right)-y_{0},
$$

where

$$
\lambda:=\frac{y_{1}-y_{0}}{x_{1}-x_{0}}
$$

- If $P=Q$, i.e., $x_{0}=x_{1}$ and $y_{0}=y_{1}$, then

$$
x_{2}=\lambda^{2}-2 x_{0}, \quad y_{2}=3 x_{0} \lambda-\lambda^{3}-y_{0},
$$

where

$$
\lambda:=\frac{3 x_{0}^{2}-A^{2}}{2 y_{0}} .
$$

Below we shall write $2 * P$ instead of $P+P$. 
- If $x_{0}=x_{1}$ and $y_{0}=-y_{1}$, then $P+Q:=\mathscr{O}$. In particular, $(0,0)+(0,0)=(A, 0)+(A, 0)=$ $(-A, 0)+(-A, 0)=\mathscr{O}$.

- Finally, we define $\mathscr{O}+P:=P$ and $P+\mathscr{O}:=P$ for any point $P$, in particular, $\mathscr{O}+\mathscr{O}=\mathscr{O}$.

With the operation "+", $\left(C_{A},+\right)$ is an abelian group with neutral element $\mathscr{O}$. Let $C_{A}(\mathbb{Q})$ be the set of rational points on $C_{A}$ together with $\mathscr{O}$. It is easy to see that $\left(C_{A}(\mathbb{Q}),+\right)$. is a subgroup of $\left(C_{A},+\right)$. Moreover, it is well known that the group $\left(C_{A}(\mathbb{Q}),+\right)$ is finitely generated. One can readily check that the three points $(0,0)$ and $( \pm A, 0)$ are the only points on $C_{A}$ of order 2 , and one easily finds other points of finite order on $C_{A}$. But do we find also rational points of finite order on $C_{A}$ ? This question is answered by the following

Theorem 1. If $A$ is a congruent number and $\left(x_{0}, y_{0}\right)$ is a rational point on $C_{A}$ with $y_{0} \neq 0$, then the order of $\left(x_{0}, y_{0}\right)$ is infinite. In particular, if there exists one rational Pythagorean A-triple, then there exist infinitely many such triples.

The usual proofs of Theorem 1 are quite involved. For example, Koblitz [Kob93, Ch.I, §9, Prop. 17] gives a proof using Dirichlet's theorem on primes in an arithmetic progression, and in Chahal [Cha06, Thm.3], a proof is given using the Lutz-Nagell theorem, which states that rational points of finite order are integral. However, both results, Dirichlet's theorem and the Lutz-Nagell theorem, are quite deep results, and the aim of this article is to provide a simple proof of Theorem 1 which relies on an elementary theorem of Fermat.

\section{A Theorem of Fermat}

In [Fer1670], Fermat gives an algorithm to construct different right-angled triangles with three rational sides having the same area (see also Hungerbühler [Hun96]). Moreover, Fermat claims that his algorithm yields infinitely many distinct such right-angled triangles. However, he did not provide a proof for this claim. In this section, we first present Fermat's algorithm and then we show that this algorithm delivers infinitely many pairwise distinct rational right-angled triangles of the same area.

Fermat's Algorithm 2. Assume that $A$ is a congruent number, and that $\left(a_{0}, b_{0}, c_{0}\right)$ is a rational Pythagorean A-triple, i.e., $A=\left|\frac{a_{0} b_{0}}{2}\right|$. Then

$$
a_{1}:=\frac{4 c_{0}^{2} a_{0} b_{0}}{2 c_{0}\left(a_{0}^{2}-b_{0}^{2}\right)}, \quad b_{1}:=\frac{c_{0}^{4}-4 a_{0}^{2} b_{0}^{2}}{2 c_{0}\left(a_{0}^{2}-b_{0}^{2}\right)}, \quad c_{1}:=\frac{c_{0}^{4}+4 a_{0}^{2} b_{0}^{2}}{2 c_{0}\left(a_{0}^{2}-b_{0}^{2}\right)},
$$

is also a rational Pythagorean A-triple. Moreover, $a_{0} b_{0}=a_{1} b_{1}$, i.e., if $\left(a_{0}, b_{0}, c_{0}, A\right) \in P(\mathbb{Q})$, then $\left(a_{1}, b_{1}, c_{1}, A\right) \in P(\mathbb{Q})$.

Proof. Let $m:=c_{0}^{2}$, let $n:=2 a_{0} b_{0}$, and let

$$
X:=2 m n, \quad Y:=m^{2}-n^{2}, \quad Z:=m^{2}+n^{2},
$$

in other words,

$$
X=4 c_{0}^{2} a_{0} b_{0}, \quad Y=c_{0}^{4}-4 a_{0}^{2} b_{0}^{2}, \quad Z=c_{0}^{4}+4 a_{0}^{2} b_{0}^{2} .
$$

Then obviously, $X^{2}+Y^{2}=Z^{2}$, and since $a_{0}, b_{0}, c_{0} \in \mathbb{Q},(|X|,|Y|,|Z|)$ is a rational Pythagorean triple, where the area of the corresponding right-angled triangle is

$$
\tilde{A}=\left|\frac{X Y}{2}\right|=\left|2 a_{0} b_{0} c_{0}^{2}\left(c_{0}^{4}-4 a_{0}^{2} b_{0}^{2}\right)\right| \text {. }
$$

Since $a_{0}^{2}+b_{0}^{2}=c_{0}^{2}$, we get $c_{0}^{4}=\left(a_{0}^{2}+b_{0}^{2}\right)^{2}=a_{0}^{4}+2 a_{0}^{2} b_{0}^{2}+b_{0}^{4}$ and therefore

$$
c_{0}^{4}-4 a_{0}^{2} b_{0}^{2}=a_{0}^{4}-2 a_{0}^{2} b_{0}^{2}+b_{0}^{4}=\left(a_{0}^{2}-b_{0}^{2}\right)^{2}>0 .
$$


So, for

$$
a_{1}=\frac{X}{2 c_{0}\left(a_{0}^{2}-b_{0}^{2}\right)}, \quad b_{1}=\frac{Y}{2 c_{0}\left(a_{0}^{2}-b_{0}^{2}\right)}, \quad c_{1}=\frac{Z}{2 c_{0}\left(a_{0}^{2}-b_{0}^{2}\right)},
$$

we have $a_{1}^{2}+b_{1}^{2}=c_{1}^{2}$ and

$$
\frac{a_{1} b_{1}}{2}=\frac{X Y}{2 \cdot 4 c_{0}^{2}\left(a_{0}^{2}-b_{0}^{2}\right)^{2}}=\frac{2 a_{0} b_{0} c_{0}^{2}\left(c_{0}^{4}-4 a_{0}^{2} b_{0}^{2}\right)}{4 c_{0}^{2}\left(a_{0}^{2}-b_{0}^{2}\right)^{2}}=\frac{2 a_{0} b_{0} c_{0}^{2}\left(a_{0}^{2}-b_{0}^{2}\right)^{2}}{4 c_{0}^{2}\left(a_{0}^{2}-b_{0}^{2}\right)^{2}}=\frac{a_{0} b_{0}}{2} .
$$

Theorem 3. Assume that $A$ is a congruent number, that $\left(a_{0}, b_{0}, c_{0}\right)$ is a rational Pythagorean $A$ triple, and for positive integers $n$, let $\left(a_{n}, b_{n}, c_{n}\right)$ be the rational Pythagorean A-triple we obtain by Fermat's Algorithm from $\left(a_{n-1}, b_{n-1}, c_{n-1}\right)$. Then for any distinct non-negative integers $n, n^{\prime}$, we have $\left|c_{n}\right| \neq\left|c_{n^{\prime}}\right|$.

Proof. Let $n$ be an arbitrary but fixed non-negative integer. Since $A=\left|\frac{a_{n} b_{n}}{2}\right|$, we have $2 A=\left|a_{n} b_{n}\right|$, and consequently

$$
a_{n}^{2} b_{n}^{2}=4 A^{2}
$$

Furthermore, since $a_{n}^{2}+b_{n}^{2}=c_{n}^{2}$, we have

$$
\left(a_{n}^{2}+b_{n}^{2}\right)^{2}=a_{n}^{4}+2 a_{n}^{2} b_{n}^{2}+b_{n}^{4}=a_{n}^{4}+8 A^{2}+b_{n}^{4}=c_{n}^{4},
$$

and consequently we get

$$
c_{n}^{4}-16 A^{2}=a_{n}^{4}-8 A^{2}+b_{n}^{4}=a_{n}^{4}-2 a_{n}^{2} b_{n}^{2}+b_{n}^{4}=\left(a_{n}^{2}-b_{n}^{2}\right)^{2}>0 .
$$

Therefore,

$$
\sqrt{\left(a_{n}^{2}-b_{n}^{2}\right)^{2}}=\left|a_{n}^{2}-b_{n}^{2}\right|=\sqrt{c_{n}^{4}-16 A^{2}},
$$

and with (2.5) and (2.6) we finally have

$$
\left|c_{n+1}\right|=\frac{c_{n}^{4}+16 A^{2}}{2 c_{n} \sqrt{c_{n}^{4}-16 A^{2}}} .
$$

Now, assume that $c_{n}=\frac{u}{v}$ where $u$ and $v$ are in lowest terms. We consider the following two cases:

$u$ is odd: First, we write $v=2^{k} \cdot \tilde{v}$, where $k \geq 0$ and $\tilde{v}$ is odd. In particular, $c_{n}=\frac{u}{2^{k} \cdot \tilde{v}}$. Since $c_{n+1}$ is rational, $\sqrt{c_{n}^{4}-16 A^{2}} \in \mathbb{Q}$. So,

$$
\sqrt{c_{n}^{4}-16 A^{2}}=\sqrt{\frac{u^{4}-16 A^{2} v^{4}}{v^{4}}}=\frac{\tilde{u}}{v^{2}}
$$

for a positive odd integer $\tilde{u}$. Then

$$
\left|c_{n+1}\right|=\frac{\frac{u^{4}+16 A^{2} v^{4}}{v^{4}}}{\frac{2 u \tilde{u}}{v^{3}}}=\frac{\bar{u}}{2 u \tilde{u} v}=\frac{\bar{u}}{2 u \tilde{u} 2^{k} \tilde{v}}=\frac{\bar{u}}{2^{k+1} u \tilde{u} \tilde{v}}=\frac{u^{\prime}}{2^{k+1} \cdot v^{\prime}}
$$

where $\bar{u}, u^{\prime}, v^{\prime}$ are odd integers and $\operatorname{gcd}\left(u^{\prime}, v^{\prime}\right)=1$. This shows that

$$
c_{n}=\frac{u}{2^{k} \cdot \tilde{v}} \Rightarrow\left|c_{n+1}\right|=\frac{u^{\prime}}{2^{k+1} \cdot v^{\prime}}
$$

where $u, \tilde{v}, u^{\prime}, v^{\prime}$ are odd. 
$u$ is even: First, we write $u=2^{k} \cdot \tilde{u}$, where $k \geq 1$ and $\tilde{u}$ is odd. In particular, $c_{n}=\frac{2^{k} \cdot \tilde{u}}{v}$, where $v$ is odd. Similarly, $A=2^{l} \cdot \tilde{A}$, where $l \geq 0$ and $\tilde{A}$ is odd. Then

$$
c_{n}^{4} \pm 16 A^{2}=\frac{2^{4 k} \cdot \tilde{u}^{4} \pm 2^{4+2 l} \tilde{A}^{2} v^{4}}{v^{4}},
$$

where both numbers are of the form

$$
\frac{2^{2 m} \bar{u}}{v^{4}}
$$

where $\bar{u}$ is odd and $4 \leq 2 m \leq 4 k$, i.e., $2 \leq m \leq 2 k$. Therefore,

$$
\left|c_{n+1}\right|=\frac{2^{2 m} u_{0} \cdot v^{3}}{2 \cdot 2^{k} \tilde{u} \cdot v^{4} \cdot 2^{m} u_{1}}=\frac{2^{m-k-1} \cdot u^{\prime}}{v^{\prime}}
$$

where $u_{0}, u_{1}, u^{\prime}, v^{\prime}$ are odd. Since $m<2 k+1$, we have $m-k-1<k$, and therefore we obtain

$$
c_{n}=\frac{2^{k} \cdot \tilde{u}}{v} \Rightarrow\left|c_{n+1}\right|=\frac{2^{k^{\prime}} \cdot u^{\prime}}{v^{\prime}}
$$

where $\tilde{u}, v, u^{\prime}, v^{\prime}$ are odd and $0 \leq k^{\prime}<k$.

Both cases together show that whenever $c_{n}=2^{k} \cdot \frac{u}{v}$, where $k \in \mathbb{Z}$ and $u, v$ are odd, then $\left|c_{n+1}\right|=$ $2^{k^{\prime}} \cdot \frac{u^{\prime}}{v^{\prime}}$, where $u^{\prime}, v^{\prime}$ are odd and $k^{\prime}<k$. So, for any distinct non-negative integers $n$ and $n^{\prime},\left|c_{n}\right| \neq$ $\left|c_{n+1}\right|$.

The proof of Theorem 3 gives us the following reformulation of Fermat's Algorithm:

Corollary 4. Assume that $A$ is a congruent number, and that $\left(a_{0}, b_{0}, c_{0}\right)$ is a rational Pythagorean A-triple, i.e., $A=\left|\frac{a_{0} b_{0}}{2}\right|$. Then

$$
a_{1}=\frac{4 A c_{0}}{\sqrt{c_{0}^{4}-16 A^{2}}}, \quad b_{1}=\frac{\sqrt{c_{0}^{4}-16 A^{2}}}{2 c_{0}}, \quad c_{1}=\frac{c_{0}^{4}+16 A^{2}}{2 c_{0} \sqrt{c_{0}^{4}-16 A^{2}}},
$$

is also a rational Pythagorean A-triple.

Proof. Notice that $c_{0}^{4}-4 a_{0}^{2} b_{0}^{2}=c_{0}^{4}-16 A^{2}$ and recall that $\left|a_{0}^{2}-b_{0}^{2}\right|=\sqrt{c_{0}^{4}-16 A^{2}}$.

\section{Doubling points with Fermat's Algorithm}

Before we prove Theorem 1 (i.e., that congruent number curves do not contain rational points of finite order), we first prove that Fermat's Algorithm 2 is essentially doubling points on congruent number curves.

Lemma 5. Let $A$ be a congruent number, let $\left(a_{0}, b_{0}, c_{0}\right)$ be a rational Pythagorean $A$-triple, and let $\left(a_{1}, b_{1}, c_{1}\right)$ be the rational Pythagorean A-triple obtained by Fermat's Algorithm from $\left(a_{0}, b_{0}, c_{0}\right)$. Furthermore, let $\left(x_{0}, y_{0}\right)$ and $\left(x_{1}, y_{1}\right)$ be the rational points on the curve $C_{A}$ which correspond to $\left(a_{0}, b_{0}, c_{0}\right)$ and $\left(a_{1}, b_{1}, c_{1}\right)$, respectively. Then we have

$$
2 *\left(x_{0}, y_{0}\right)=\left(x_{1},-y_{1}\right) .
$$

Proof. Let $\left(a_{0}, b_{0}, c_{0}\right)$ be a rational Pythagorean $A$-triple. Then, according to (2.5), the rational Pythagorean $A$-triple $\left(a_{1}, b_{1}, c_{1}\right)$ which we obtain by Fermat's Algorithm is given by

$$
a_{1}:=\frac{4 c_{0}^{2} a_{0} b_{0}}{2 c_{0}\left(a_{0}^{2}-b_{0}^{2}\right)}, \quad b_{1}:=\frac{c_{0}^{4}-4 a_{0}^{2} b_{0}^{2}}{2 c_{0}\left(a_{0}^{2}-b_{0}^{2}\right)}, \quad c_{1}:=\frac{c_{0}^{4}+4 a_{0}^{2} b_{0}^{2}}{2 c_{0}\left(a_{0}^{2}-b_{0}^{2}\right)} .
$$


Now, by (1.1), the coordinates of the rational point $\left(x_{1}, y_{1}\right)$ on $C_{A}$ which corresponds to the rational Pythagorean $A$-triple $\left(a_{1}, b_{1}, c_{1}\right)$ are given by

$$
\begin{aligned}
& x_{1}=\frac{a_{0} b_{0} \cdot\left(b_{1}+c_{1}\right)}{2 \cdot a_{1}}=\frac{a_{0} b_{0} \cdot 2 c_{0}^{4}}{2 \cdot 4 c_{0}^{2} a_{0} b_{0}}=\frac{c_{0}^{2}}{4}, \\
& y_{1}=\frac{2\left(\frac{a_{0} b_{0}}{2}\right)^{2}\left(b_{1}+c_{1}\right)}{a_{1}^{2}}=\frac{1}{8}\left(a_{0}^{2}-b_{0}^{2}\right) c_{0} .
\end{aligned}
$$

Let still $\left(a_{0}, b_{0}, c_{0}\right)$ be a rational Pythagorean $A$-triple. Then, again by (1.1), the corresponding rational point $\left(x_{0}, y_{0}\right)$ on $C_{A}$ is given by

$$
x_{0}=\frac{b_{0}\left(b_{0}+c_{0}\right)}{2}, \quad y_{0}=\frac{b_{0}^{2}\left(b_{0}+c_{0}\right)}{2} .
$$

Now, as we have seen in (1.3) and (1.4), the coordinates of the point $\left(x_{1}^{\prime}, y_{1}^{\prime}\right):=2 *\left(x_{0}, y_{0}\right)$ are given by $x_{1}^{\prime}=\lambda^{2}-2 x_{0}, y_{1}^{\prime}=3 x_{0} \lambda-\lambda^{3}-y_{0}$, where

$$
\begin{aligned}
\lambda=\frac{3 x_{0}^{2}-\left(\frac{a_{0} b_{0}}{2}\right)^{2}}{2 y_{0}}=\frac{\frac{3 b_{0}^{2}\left(b_{0}+c_{0}\right)^{2}-a_{0}^{2} b_{0}^{2}}{4}}{b_{0}^{2}\left(b_{0}+c_{0}\right)}=\frac{3\left(b_{0}+c_{0}\right)^{2}-a_{0}^{2}}{4\left(b_{0}+c_{0}\right)}=\frac{3\left(b_{0}+c_{0}\right)^{2}+\left(b_{0}^{2}-c_{0}^{2}\right)}{4\left(b_{0}+c_{0}\right)}= \\
\frac{\left(3 b_{0}^{2}+6 b_{0} c_{0}+3 c_{0}^{2}\right)+\left(b_{0}^{2}-c_{0}^{2}\right)}{4\left(b_{0}+c_{0}\right)}=\frac{4 b_{0}^{2}+6 b_{0} c_{0}+2 c_{0}^{2}}{4\left(b_{0}+c_{0}\right)}=\frac{2 b_{0}^{2}+3 b_{0} c_{0}+c_{0}^{2}}{2\left(b_{0}+c_{0}\right)}= \\
\frac{\left(2 b_{0}+c_{0}\right)\left(b_{0}+c_{0}\right)}{2\left(b_{0}+c_{0}\right)}=\frac{\left(2 b_{0}+c_{0}\right)}{2} .
\end{aligned}
$$

Hence,

$$
x_{1}^{\prime}=\lambda^{2}-2 x_{0}=\frac{\left(2 b_{0}+c_{0}\right)^{2}}{4}-b_{0}\left(b_{0}+c_{0}\right)=\frac{\left(4 b_{0}^{2}+4 b_{0} c_{0}+c_{0}^{2}\right)-\left(4 b_{0}^{2}+4 b_{0} c_{0}\right)}{4}=\frac{c_{0}^{2}}{4}
$$

and

$$
y_{1}^{\prime}=3 x_{0} \lambda-\lambda^{3}-y_{0}=\frac{1}{8}\left(2 b_{0}^{2} c_{0}-c_{0}^{3}\right)=\frac{1}{8}\left(b_{0}^{2}-a_{0}^{2}\right) c_{0},
$$

i.e., $x_{1}=x_{1}^{\prime}$ and $y_{1}=-y_{1}^{\prime}$, as claimed.

With Lemma 5, we are now able to prove Theorem 1, which states that for a congruent number $A$, the curve $C_{A}: y^{2}=x^{3}-A^{2} x$ does not have rational points of finite order other than $(0,0)$ and $( \pm A, 0)$.

Proof of Theorem 1. Assume that $A$ is a congruent number, let $\left(x_{0}, y_{0}\right)$ be a rational point on $C_{A}$ which $y_{0} \neq 0$, and let $\left(a_{0}, b_{0}, c_{0}\right)$ be the rational Pythagorean $A$-triple which corresponds to $\left(x_{0}, y_{0}\right)$ by (1.2). Furthermore, for positive integers $n$, let $\left(a_{n}, b_{n}, c_{n}\right)$ be the rational Pythagorean $A$-triple we obtain by Fermat's Algorithm from $\left(a_{n-1}, b_{n-1}, c_{n-1}\right)$, and let $\left(x_{n}, y_{n}\right)$ be the rational point on $C_{A}$ which corresponds to the rational Pythagorean $A$-triple $\left(a_{n}, b_{n}, c_{n}\right)$ by (1.1).

By the proof of Lemma 5 we know that the $x$-coordinate of $2 *\left(x_{n}, y_{n}\right)$ is equal to $\frac{c_{n}^{2}}{4}$, and by Theorem 3 we have that for any distinct non-negative integers $n, n^{\prime},\left|c_{n}\right| \neq\left|c_{n^{\prime}}\right|$. Hence, for all distinct non-negative integers $n, n^{\prime}$ we have

$$
\left(x_{n}, y_{n}\right) \neq\left(x_{n^{\prime}}, y_{n^{\prime}}\right)
$$

which shows that the order of $\left(x_{0}, y_{0}\right)$ is infinite. 


\section{References}

[Cha06] Jasbir S. Chahal, Congruent numbers and elliptic curves, American Mathematical Monthly 113 (2006), $308-317$.

[Fer1670] Pierre de Fermat, Fermat's Diophanti Alex. Arith., 1670 in Euvres III (Ministère de l'instruction publique, ed.), Gauthier-Villars et fils, Paris, 1896, pp. 254-256.

[Hun96] Norbert Hungerbühler, A proof of a conjecture of Lewis Carroll, Mathematics Magazine, 69 (1996), $182-184$.

[Kob93] Neal Koblitz, Introduction to Elliptic Curves and Modular Forms, 2nd edition, Graduate Texts in Mathematics 97, Springer-Verlag, New York, 1993.

\section{Lorenz Halbeisen}

Department of Mathematics

ETH Zentrum, Rämistrasse 101

8092 Zürich, Switzerland

e-mail: lorenz.halbeisen@math.ethz.ch

\section{Norbert Hungerbühler}

Department of Mathematics

ETH Zentrum, Rämistrasse 101

8092 Zürich, Switzerland

e-mail: norbert.hungerbuehler@math.ethz.ch 